\title{
NDC ME (Milligram)
}

National Cancer Institute

\section{Source}

National Cancer Institute. NDC ME (Milligram). NCI Thesaurus. Code C155838.

A recognized billing qualifier that may be used to identify milligrams as the NDC unit of measure. 